\title{
Numerical Simulation of Fault Zone Guided Waves: Accuracy and 3-D Effects
}

\author{
Heiner Igel, ${ }^{1}$ Gunnar Jahnke, ${ }^{1}$ and Yehuda Ben-Zion ${ }^{2}$
}

\begin{abstract}
Fault zones are thought to consist of regions with reduced seismic velocity. When sources are located in or close to these low-velocity zones, guided seismic head and trapped waves are generated which may be indicative of the structure of fault zones at depth. Observations above several fault zones suggest that they are common features of near fault radiation, yet their interpretation may be highly ambiguous. Analytical methods have been developed to calculate synthetic seismograms for sources in fault zones as well as at the material discontinuities. These solutions can be used for accurate modeling of wave propagation in plane-parallel layered fault zone structures. However, at present it is not clear how modest deviations from such simplified geometries affect the generation efficiency and observations of trapped wave motion. As more complicated models cannot be solved by analytical means, numerical methods must be employed. In this paper we discuss 3-D finite-difference calculations of waves in modestly irregular fault zone structures. We investigate the accuracy of the numerical solutions for sources at material interfaces and discuss some dominant effects of 3-D structures. We also show that simple mathematical operations on 2-D solutions generated with line sources allow accurate modeling of 3-D wave propagation produced by point sources. The discussed simulations indicate that structural discontinuities of the fault zone (e.g., fault offsets) larger than the fault zone width affect significantly the trapping efficiency, while vertical properly gradients, fault zone narrowing with depth, small-scale structures, and moderate geometrical variations do not. The results also show that sources located with appropriate orientations outside and below a shallow fault zone layer can produce considerable guided wave energy in the overlying fault zone layer.
\end{abstract}

Key words: Fault zones, guided waves, finite differences.

\section{Introduction}

Fault zone structures are thought to contain a highly damaged material having lower seismic velocity than the surrounding rocks. If the highly damaged fault zone material is spatially coherent it can act as a waveguide for seismic fault zone head and trapped waves. Fault zone head waves propagate along material discontinuity interfaces in the structure, while trapped waves are critically reflected phases

\footnotetext{
1 Institut für Geophysik, Ludwig-Maximilians-Universität, Theresienstrasse 41, 80333 Munich, Germany. E-mails: igel@geophysik.uni-muenchen.de; jahnke@geophysik.uni-muenchen.de

${ }^{2}$ Department of Earth Sciences, University of Sourthern California, Los Angeles, U.S.A. E-mail: benzion@terra.usc.edu

Corresponding author: Prof. Dr. Heiner Igel
} 
traveling inside low velocity fault zone layers with dispersive character (BEN-ZION and AKI, 1990; BEN-ZION, 1998). Seismic fault zone waves have been observed above several major faults (e.g., subduction zone of the Philippine Sea plate underneath Japan, the San Andreas Fault, rupture zones of the Kobe, Japan, and Landers, CA, eathquakes) and imaged by several authors with the major conclusion that structures can be recovered at a resolution of a few tens of meters (e.g., FUKAO et al., 1983; LEARY et al., 1987; Li et al., 1990, 1994a, b, 1999; BEN-Zion and MALin, 1991; BENZion et al., 1992; Hough et al., 1994; Michael and Ben-Zion, 2001).

As details of the fault zone structure may have important implications for the stress build-up and release, there is considerable interest in devising reliable means to image fault zone structure at depth. Fox example, structural (dis-)continuities of fault zones may dominantly affect the size of likely ruptures and thus the magnitude of future earthquakes. From an observational point of view more and more aftershock regions of large earthquakes are monitored with dense networks of mobile seismometers. These networks combined with increased seismicity in such circumstances offer unique opportunities to collect large data sets and estimate the in situ structure of the associated regions. Accurate determination of fault zone structure also has implications for earthquake location, focal mechanisms and estimates of pre-, co- and post-seismic deformation.

An important modeling tool of fault zone properties at depth can be provided by accurate simulations of seismic fault zone head and trapped waves for realisitic structures. To calculate synthetic seismograms for low velocity structures several approaches can be taken: CORMIER and SPUDICH (1984), Hori et al. (1985) and CORMIER and BEROZA (1987) used ray-theory to model low-velocity zones. In a series of papers, BEN-ZION $(1989,1990,1999)$ and BEN-ZION and AKI (1990) developed 2-D and 3-D analytical solutions for seismic wavefields generated by double-couple sources at material discontinuities in plane-parallel structures. Extensive 2-D studies of the dependency of fault zone wave motion on basic media properties and sourcereceiver geometries (e.g., BEN-Zion, 1998; MiChaEL and BEN-Zion, 1998) show that there are significant trade-offs between propagation distance along the structure, fault zone width, impedance contrasts, source location within the FZ, and $Q$. These trade-offs and additional sources of uncertainties make a reliable determination of fault zone structure a very challenging endeavor.

To date, the analysis and inversion of head and trapped waveforms, have been predominantly carried out using 2-D models (e.g., LI et al., 1994a, b; HougH et al., 1994; Michael and Ben-Zion, 1998, 2001; Peng et al., 2000), resulting in surprisingly good waveform fits. However, what aspects of the medium parameters are well resolved - particularly given the possibility of 3-D structure - is difficult to judge. Correct interpretation of fault zone guided waves requires a basic understanding of the wavefield in irregular geometries. Several studies have attempted to investigate waves in fault zones with irregular structures (e.g., LEARY et al., 1991, 1993; Huang et al., 1995; Li and Vidale, 1996; Igel et al., 1997; Li et al., 1998). 
These studies were carried out using 2-D and/or acoustic approximations. A thorough phenomenological study of the effects of deviations from simple fault zone geometries should be beyond these approximations.

In this study we present numerical solutions for 3-D elastic wave propagation with fault zone structures, using a high-order finite-difference method. The main goals are (1) to verify the accuracy of the method by comparing numerical with analytical solutions, (2) to compare 2-D (line source) solutions with 3-D (point source) solutions and (3) to discuss the effects of some 3-D structures on the wavefield. A study of waves associated with a larger set of 3-D fault zone structures and detailed analysis of the waveforms are reported elsewhere (JAHNKE et al., 2001).

\section{Methods, Accuracy, Verification}

In order to calculate the seismic wavefield for irregular 3-D models, numerical solutions must be employed. Several numerical techniques are being applied to problems in exploration, regional and global seismology. These include the finitedifference method (e.g., VirieuX, 1986; Graves, 1993; Igel et al., 1995) pseudospectral methods (e.g., TesSMer, 1995; Furumura et al., 1998; IGEL, 1999), spectral elements (e.g., KomatiTsch et al., 2000), finite volumes (e.g., Dormy and TARANTOLA, 1995; KäSER et al., 2001), and coupled normal mode calculations (e.g. PolLITZ, this volume). High-order straggered-grid finite-difference methods offer a flexible approach, in particular since they are easily adapted to parallel hardware due to the local character of the differential operators. The calculations presented here are performed with a fourth-order scheme in space and second order in time. Problems with this approach may arise since the elements of stress and strain tensors as well as the components of the velocity vector are not defined at the same location due to grid staggering. This is especially relevant for general anisotropic media in which additional numerical interpolations are necessary, which in general degrade the solution (IGEL et al., 1995). Attenuation can be included in such numerical schemes using he concept of memory variations (e.g., CARCIONE et al., 1988).

Another complication may arise in connection with the specific geometries encountered in fault zones: Seismic sources are likely to occur directly at material interfaces (e.g., BEN-Zion and ANDREws, 1998; SiBSON, 1999), possibly along the sides of near-vertical low-velocity fault zone layers. Thus far analytical solutions for seismic radiation from dislocations along material interfaces (e.g., BEN-ZION, 1989, 1990) have only been matched by numerical solutions for the SH case and line sources (IGEL et al., 1997) but not for the 3-D case. Matching these solutions is a precondition for a reliable numerical modeling of wave propagation in complex fault zone structures.

The geometry of the basic model with a lateral material discontinuity is shown in Figure 1. A point source is located at $1000 \mathrm{~m}$ depth along the interface with material parameters given in terms of seismic velocities $v_{p}$ and $v_{s}$ (or Lamé parameters $\lambda$ and $\mu$ ) 


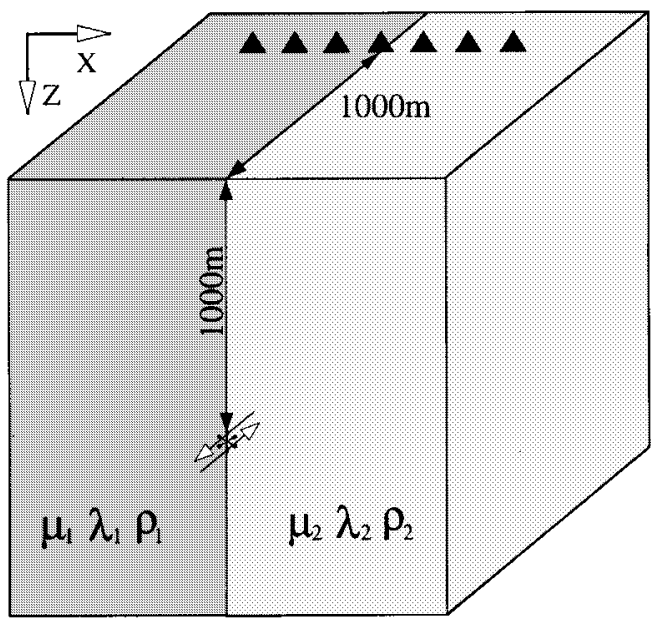

Figure 1

Model setup for the comparison with analytical solutions, the model consists of two half spaces with different seismic properties. The source is situated at the boundary of the half spaces and head waves are generated in addition to direct $P$ and $S$ waves. The source is a double-couple and the source time function is a ramp of $0.1 \mathrm{~s}$ duration.

and density $\rho$. A receiver string is located on the free surface across the material discontinuity at a horizontal distance of $1000 \mathrm{~m}$ from the source. The source is a double-couple with the only non-zero moment tensor component $M_{x y}=M_{y x}=M_{0}$ situated directly at the material interface. BEN-Zion $(1990,1999)$ showed that the resulting head waves and interface waves strongly distort the near-field waveform from corresponding waveforms in a homogeneous full space. As sources at material interfaces are realistic scenarios it is important to take into account such effects and to accurately model them with numerical techniques. The material parameters for the comparison are identical to those used by BEN-Zion (1990, 1999): In the left half space $v_{p}=5 \mathrm{~km} / \mathrm{s}, v_{s}=3.1 \mathrm{~km} / \mathrm{s}$, and $\rho=2.35 \mathrm{~g} / \mathrm{cm}^{3}$. In the right half space $v_{p}=$ $3.5 \mathrm{~km} / \mathrm{s}, v_{s}=2.17 \mathrm{~km} / \mathrm{s}$, and $\rho=1.64 \mathrm{~g} / \mathrm{cm}^{3}$. The moment source time function is a ramp of duration $0.1 \mathrm{~s}$.

The elastodynamic equations are implemented as a first-order velocity-stress system as introduced in 2-D by VIRIEUX (1986) and frequently used in combination with other numerical methods (e.g., Tessmer, 1995; IGEL, 1999; KäSER and IGEL, 2001), The resulting spatial grid is shown in Figure 2. For our particular problem, the material interface is defined in the plane $x=$ const. where the off-diagonal stress elements $\sigma_{x y}$ and the displacement components $u_{x}$ are located. The material parameters $\lambda, \mu$ and $\rho$ are also staggered. The question arises as to which parameters should be attributed to the grid points situated at the material interface, as this can dominantly influence the solution. We note that in order for the numerical solution to match the analytical solutions, the material parameters must be interpolated to the 

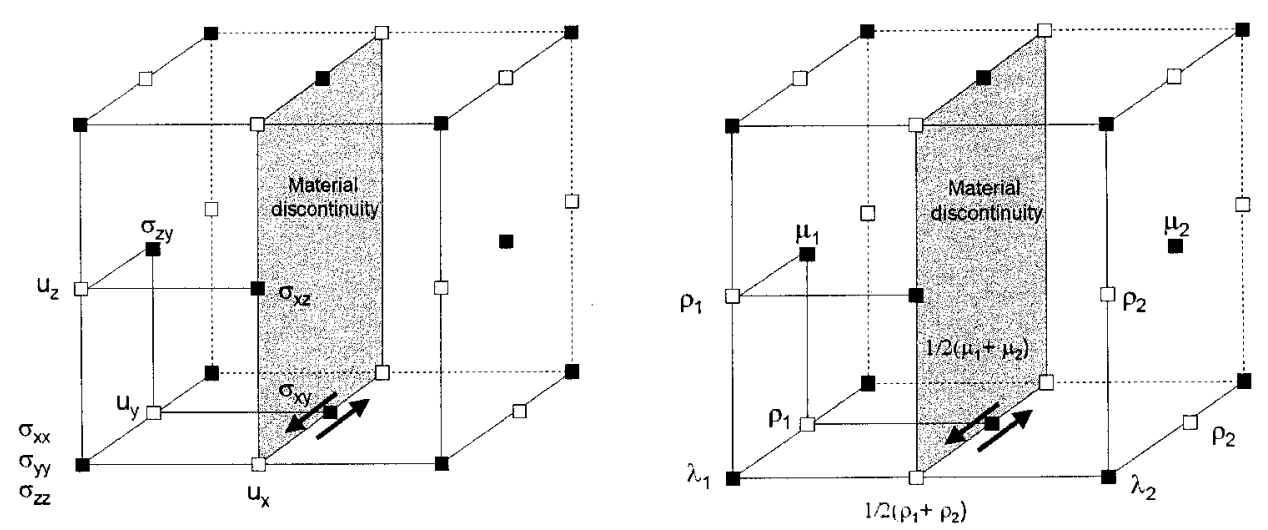

Figure 2

Left: Staggered grid used in the elastic finite-difference calculations. The material discontinuity (e.g. one side of the fault zone) is grey-shaded. The strike-slip source is located at the discontinuity and input via the moment tensor components $M_{x y}=M_{y x}=M_{0}$. The elements of one unit grid cell are annotated and connected by lines. Right: Locations where material parameters are defined. Without the interpolation of the material parameters at the interface the analytical solutions can not be accurately matched.

material interface (e.g., $\left.\mu_{i}=\left(\mu_{1}+\mu_{2}\right) / 2\right)$, where $\mu_{i}$ is the shear modulus at the interface and $\mu_{1,2}$ are the properties of the two half spaces. The same holds for the density.

Numerical and analytical displacement waveforms in 3 directions are compared in Figure 3. The grid spacing in this simulation was $25 \mathrm{~m}$. The receiver is located directly at the material interface where the effects are most prominent. The unfiltered seismograms show all the details of the analytical solution but are contaminated by numerical noise due to the discrete sampling of space and time. When the waveforms are filtered in a frequency band where the numerical method is known to be accurate, the artifacts disappear. For the example shown, the dominant frequency $(6.7 \mathrm{~Hz})$ is sampled with twenty points per wavelength for the shear waves and the root-mean square difference between analytical and numerical solution is less than $3 \%$. This indicates that the behavior of a double-couple source at a material interface is correctly modeled for the present purpose by our techniques and that wavefields in more complex models can be investigated.

\section{Geometrical Spreading, Line Sources, Corrections}

Most of the previous numerical studies of fault zone wave propagation were carried out using 2-D elastic or acoustic approximations (e.g., HUANG et al., 1995; LI et al., 1996). Furthermore, sophisticated nonlinear inversion procedures are being based on analytical solutions of trapped waves propagation for plane layered structures using line sources (e.g., Michael and Ben-Zion, 1998, 2001; Peng et al., 

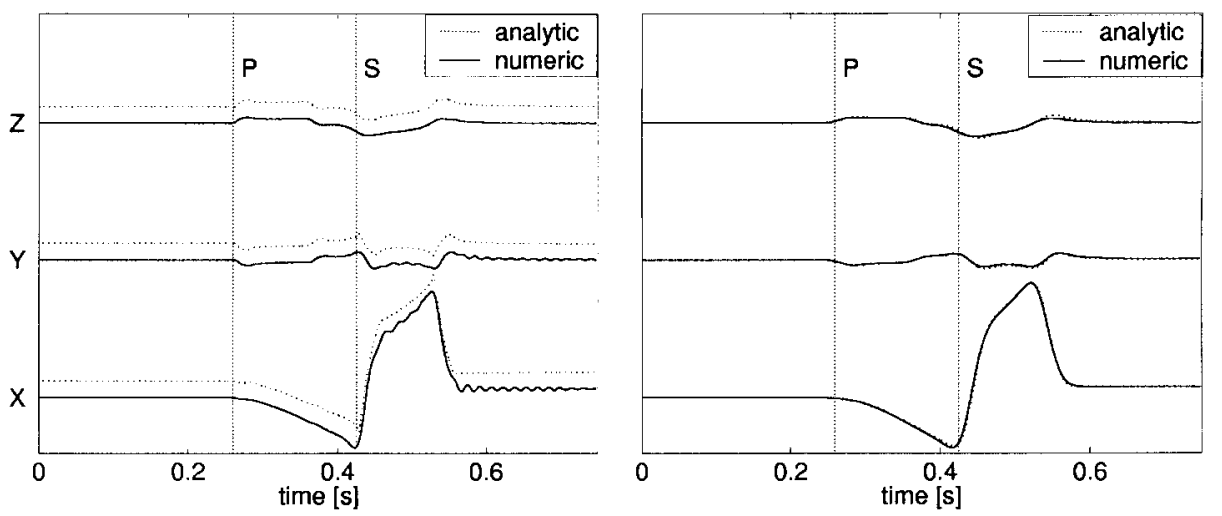

Figure 3

Left: Unfiltered seismograms of the analytical (dotted) and the numerical (solid) solution for the receiver located at the boundary of the two half spaces. For a better view the analytical solution is shifted upwards. All prominent features of the analytical wave form appear in the numerical solution. The theoretical arrival times of $P$ and $S$ waves are marked by vertical lines. Right: Filtered traces of the analytical (dotted) and the numerical (solid) solution with a dominant frequency of $6.7 \mathrm{~Hz}$. The root-mean square error of the numerical solution is less than $3 \%$.

2000). This raises the question of whether the 2-D line source solutions capture the correct behavior of 3-D point source seismograms for uniform fault zone structures. We discuss this problem using the scalar wave equation. This corresponds to SH waves with particle motion in the direction parallel to the fault zone material interfaces.

In the 2-D approximation the source is a line of infinite length. As shown by VIDALE et al. (1985), an approximated SH point source seismogram can be obtained by convolving the line source seismograms with $1 / \sqrt{t}$ and differentiating with respect to time. This is equivalent to a deconvolution with $1 / \sqrt{t}$, an approach used by other authors (e.g., CRASE et al., 1990). In previous studies the accuracy of this approximation for complicated geometries such as FZs was not discussed. Therefore we investigate here how well the point source waveforms can be reproduced by such operations on line source solutions. The applicability of the line source correction is discussed for a simple fault zone model shown in Figure 4. The source is located at the center of a fault zone of $50 \mathrm{~m}, 100 \mathrm{~m}, 150 \mathrm{~m}$ and $200 \mathrm{~m}$ width. The receiver is located at a distance of $700 \mathrm{~m}$ from the source. The dominant frequency is $15 \mathrm{~Hz}$. The properties inside the fault zone are $v_{\text {fault }}=1500 \mathrm{~m} / \mathrm{s}$ and the host rock has $v_{\text {host }}=2500 \mathrm{~m} / \mathrm{s}$.

Figure 5 shows 2-D, corrected 2-D, and 3-D seismograms recorded directly above the fault. The corrected line source seismograms (dashed lines) are almost indistinguishable from the 3-D seismograms for the examined cases. The 2-D seismograms have considerably different amplitude behavior. In addition we compare the ratio of the maximum trace amplitude with the maximum fault zone 


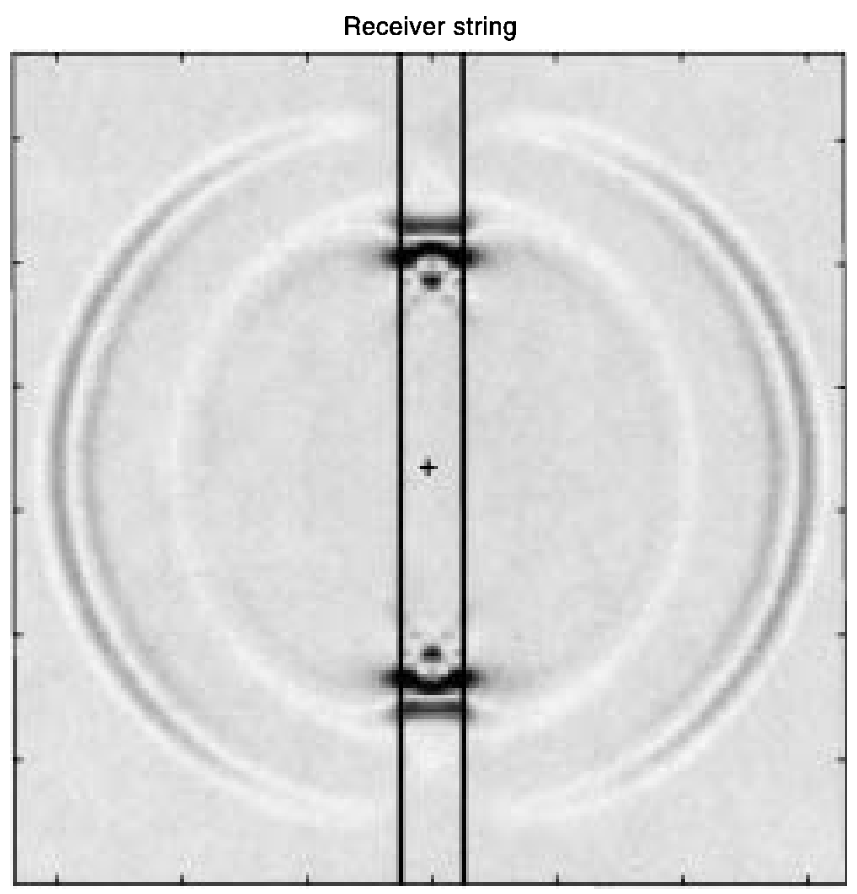

Figure 4

Snapshot of trapped wave propagation using the scalar wave equation. The fault zone width is $100 \mathrm{~m}$. The dominant frequency is $15 \mathrm{~Hz}$. The source location is indicated by a plus sign. Blue and red colors denote positive and negative amplitude, respectively. The FZ is described by 20 grid points.

wave amplitude for the different solutions. The results in Figure 6 indicate that this ratio is somewhat overestimated by the 2-D solution, and to a lesser extent also by the corrected 2-D seismograms. This should be considered when modeling real data with line source solutions.

\section{Examples: Discontinuous Fault Structures}

The modeling and interpretation of observed seismograms is usually carried out using plane layer structures (e.g., Li et al., 1994a) sometimes in combination with line dislocation sources (e.g., Hough et al., 1994; Michael and Ben-Zion, 1998; Peng et al., 2000). While it is possible to explain the observations with simple structures, the question remains how deviations from these structures would influence the wavefield. As mentioned above, the observation and interpretation of fault zone waves offer the opportunity of high resolution imaging of fault zone structure at depth. The question of resolution with respect to 3-D structure has as yet not been answered comprehensively. As important conclusions may be drawn from the 

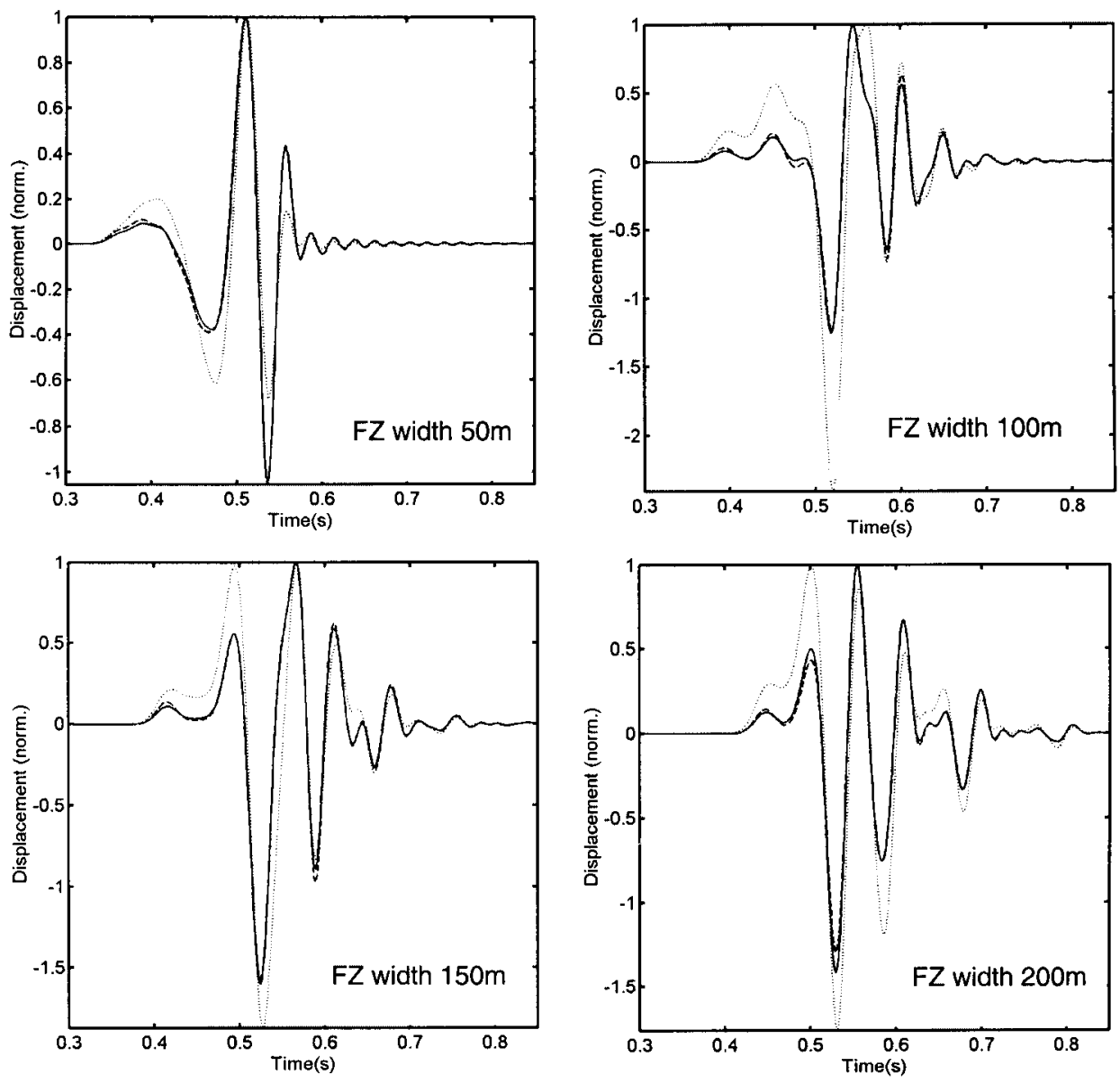

Figure 5

Comparison of 3-D (solid), 2-D (dotted) and 2-D converted (dashed) seismograms for fault zone wave propagation using the scalar wave equation (e.g. SH case) for various fault zone widths. In these cases the 2-D-3-D conversion works very well. The uncorrected 2-D seismograms have at places considerably different amplitudes.

interpretation of fault zone structure at depth, the reliability of such information is important. For example, whether fault zones are connected at depth while offset at the surface will have consequences on likely rupture geometries and size of earthquake potential on the associated structure.

Snapshots of wave propagation for a source located at the interface of a lowvelocity fault zone narrowing with depth are shown in Figure 7. The wave motion is not strongly affected by the narrowing fault; the trapped waves pass through the bottleneck and are observed as strong amplitudes at the surface. However, wave motion is considerably affected when the trapped energy impacts discontinuities 

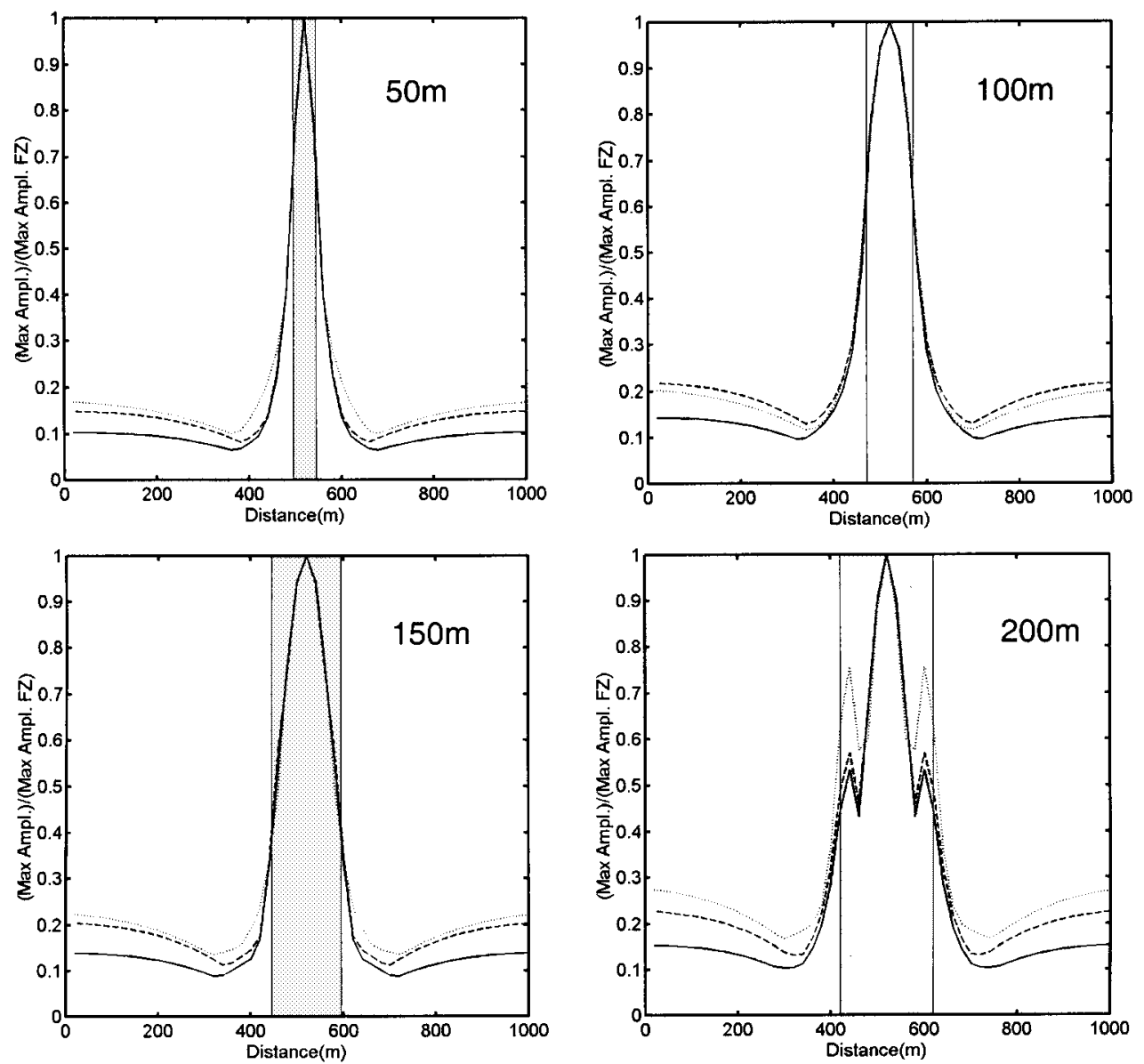

Figure 6

The variation of the ratio of maximum amplitude to fault zone wave amplitude along a profile across the fault zone for 3-D (solid), 2-D (dotted) and 2-D converted (dashed) seismograms fro different fault zone widths (same as previous Figure). The fault zone region is grey-shaded. In general the ratio of fault zone wave amplitude to body wave amplitude is overestimated outside the fault zone for 2-D and 2-D-3-D converted seismograms, using the corrections described in the text.

along the fault larger than the fault zone width. Figure 8 shows that trapped waves can propagate through an offset the size of the fault zone width. A fault zone with larger offsets (this may apply to vertical as well as near-horizontal propagation directions) may considerably affect the trapping efficiency and the observed relative amplitudes above and away from the fault. A more quantitative analysis of such models is given in a companion paper (JAHNKE et al., 2001).

In the reminder of the paper we concentrate on the following two problems: (1) Can trapped energy be generated by sources outside a fault zone? (2) Are trapped waves generated when the fault zone is connected at depth but is offset at the surface. 

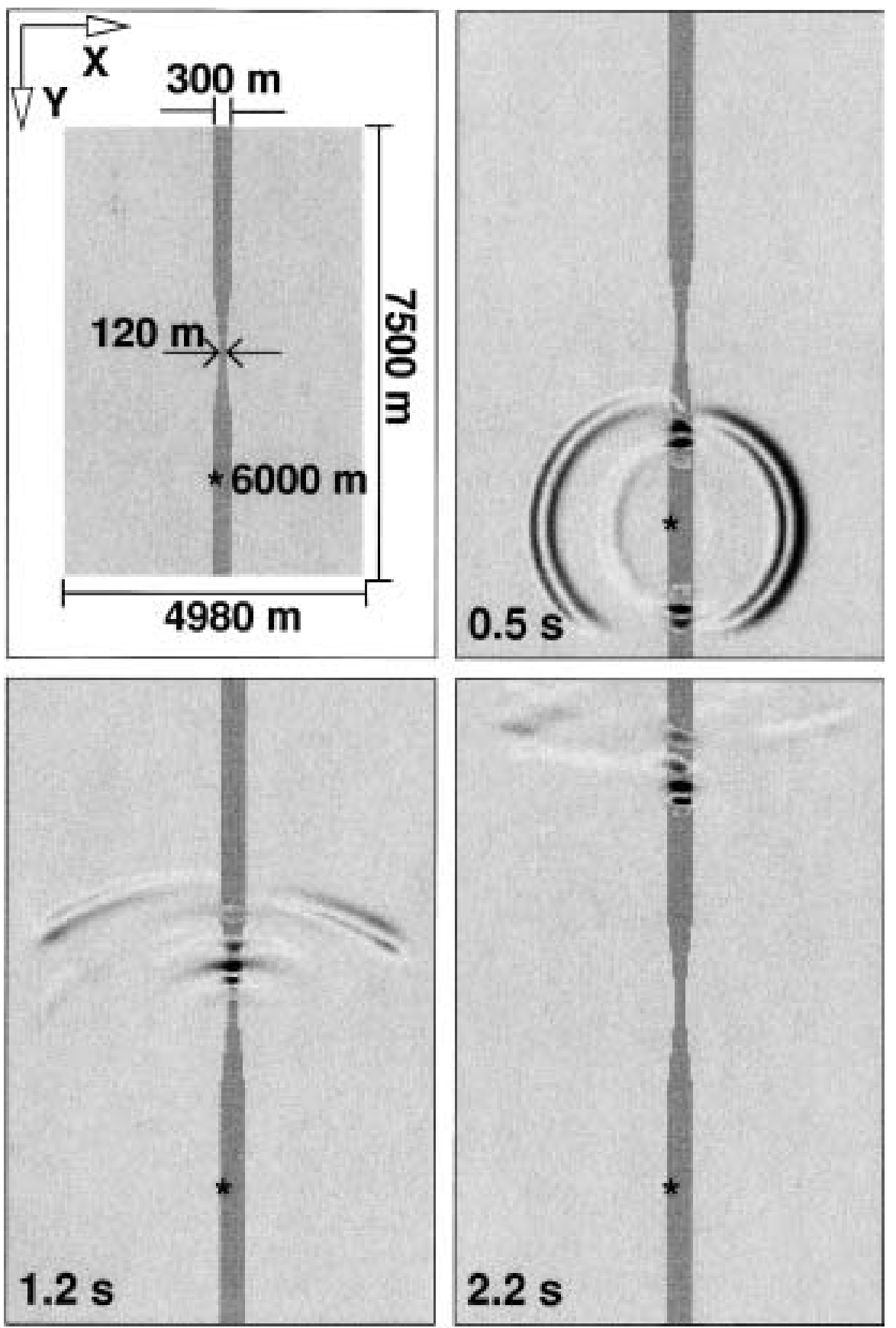

Figure 7

Snapshots of elastic wave propagation in a fault zone with bottleneck structure. Red and blue colours positive and negative velocities of $y$ component (SH type motion), respectively. The source at the fault zone boundary is indicated by a star. The second phase propagating to the left of the FZ in the top right figure is the shear wave reflected from right side of the FZ. 

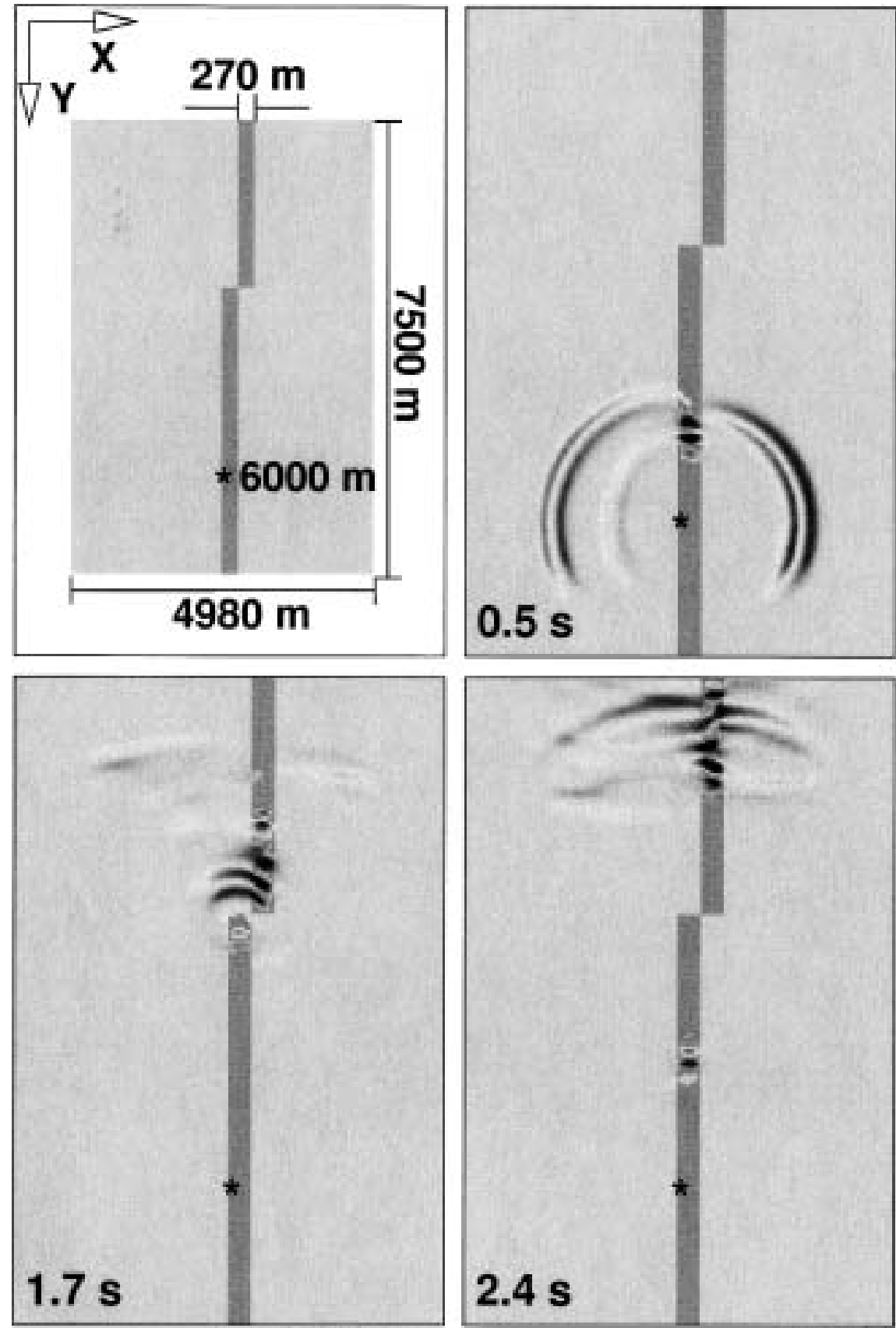

Figure 8

Snapshots of elastic wave propagation in a fault zone with offset in the vertical direction. Red and blue colors denote positive and negative velocities of $y$ component (SH type motion), respectively. The source at the fault zone boundary is indicated by a star. 
In Figure 9 three model geometries related to problem (1) are shown. The sources are located either directly below the fault zone in the host rock or below and offset by two and four fault zone widths, respectively. The source is a strike-slip dislocation with non-zero moment tensor components, $M_{x y}=M_{y x}=M_{0}$. The $y$ component of velocity is calculated for a profile across the fault directly above the source. The moment rate function is a Gaussian with a dominant frequency of $4 \mathrm{~Hz}$. The source depth is $6000 \mathrm{~m}$ and the fault zone width is $270 \mathrm{~m}$. The low-velocity fault zone layer extends from the free surface to a depth of $4000 \mathrm{~m}$. The calculations are carried out on a grid with $30 \mathrm{~m}$ grid spacing. The receiver spacing is $100 \mathrm{~m}$. With this setup, the dominant wavelength of $S$ waves within the fault zone is approximately $500 \mathrm{~m}$.

The seismograms are shown in Figure 10. Few trapped waves are generated when the source is located right below the fault zone. This is primarily because for this source-receiver geometry the fault zone is along a nodal plane of the radiated $S$ waves from the source. However, when the source is offset by several fault zone widths, sufficient energy is trapped within the low-velocity layer leading to a dominant arrival after the shear-wave onset above the fault zone. This example shows how difficult the interpretation of such arrivals can be, as the resulting signal might erroneously be mapped into fault zone parameters, assuming the structure is continuous down to source depth. This also highlights the need to combine observations from different propagation directions within the fault zone which may allow more reliable imaging of such structures.

The second example may be relevant for efforts to estimate maximum rupture sizes of future earthquakes from surface fault zone structure. Situations as shown in

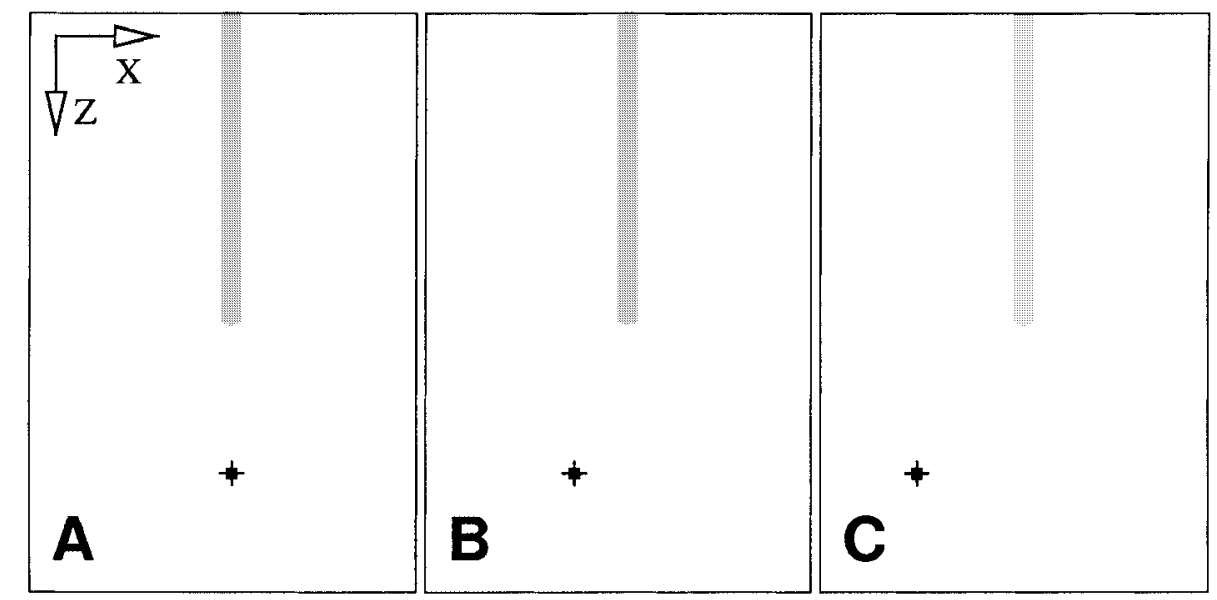

Figure 9

Three models with sources (A) below, and (B and C) below and at some distance from the fault zone. Can trapped waves be excited by such source - fault zone geometries? Is it possible to distinguish the wavefield observed for such models from models with more simple fault zone geometries? 

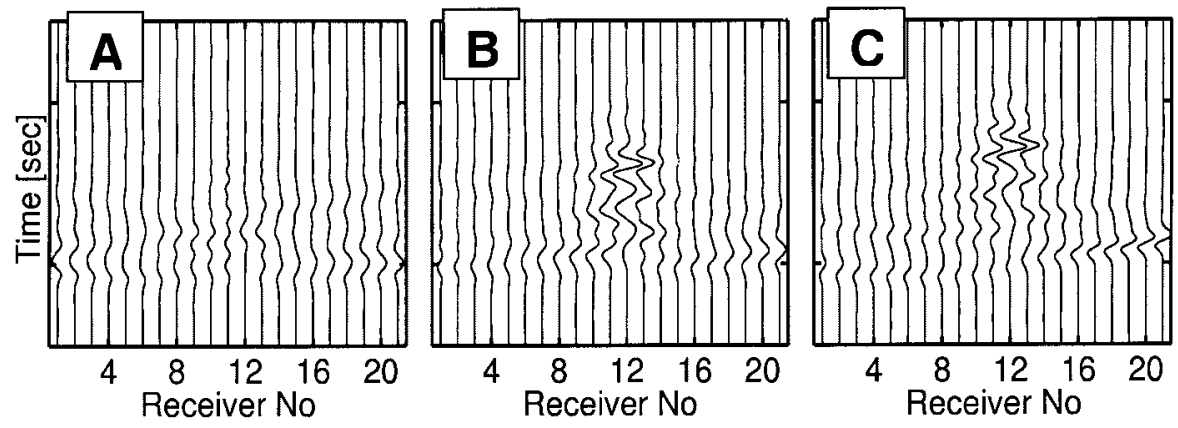

Figure 10

Horizontal component of velocity (drawn to scale) observed above the models shown in the previous Figure. Note that sources below and at some distance from the fault zone still generate trapped waves. These examples highlight the trade-off of some of the involved parameters.

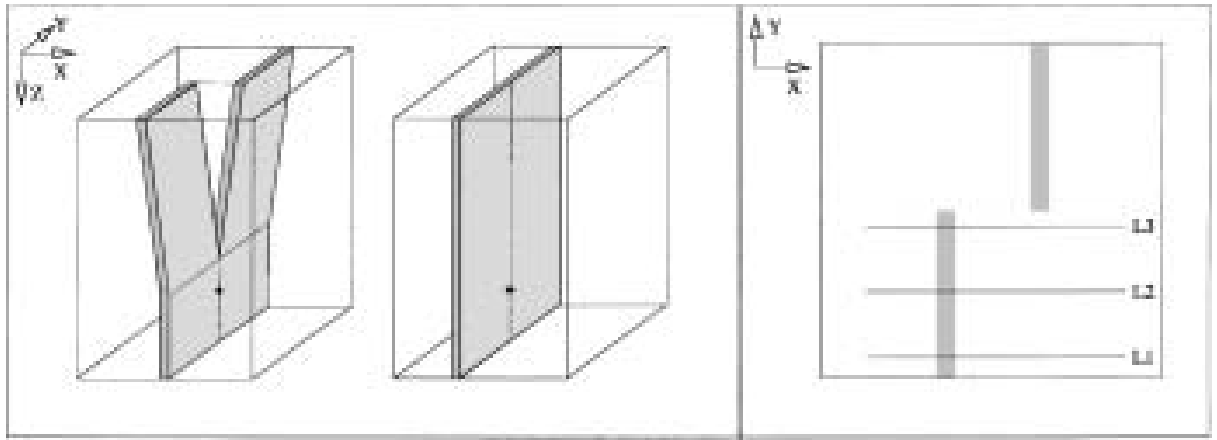

Figure 11

Left: Model with a fault zone split at the surface but connected at depth. The source location is indicated by a star. Right: Location of receiver strings above the fault zone at the surface indicated by gray shading.

Figure 11 are not unlikely: Faults are offset at the earth's surface but may be connected at depth. What are the effects of such structures on the wavefield? Is it possible to image such structures with fault zone waves? We compare seismograms for a simple fault zone with those obtained for a model with a split fault zone which is connected at depth. The wave motion is compared on three profiles (L1-3) across the fault at different locations with respect to the fault offset (Fig. 12). Trapped waves recorded above a simple fault structure remain little changed on profiles across the fault zone at moderately different offsets from the epicenter (Fig. 12, top row). However, the trapped waveforms are severely affected at some receiver lines by the splitting on the fault zone structure. The trapping efficiency for propagation directions away from the discontinuity is indistinguishable from the simple fault model. As we approach the discontinuity the trapping is severely weakened (Fig. 12, bottom right). 


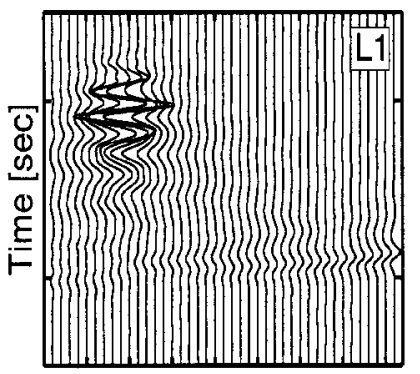

510152025303540 Receiver No

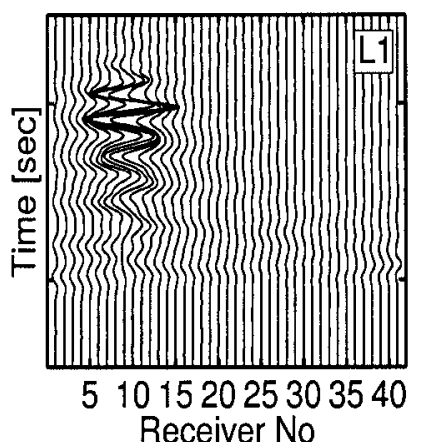

Receiver No

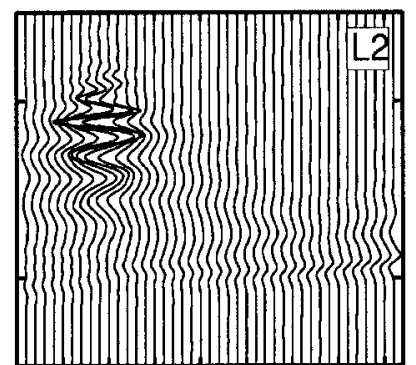

510152025303540 Receiver No

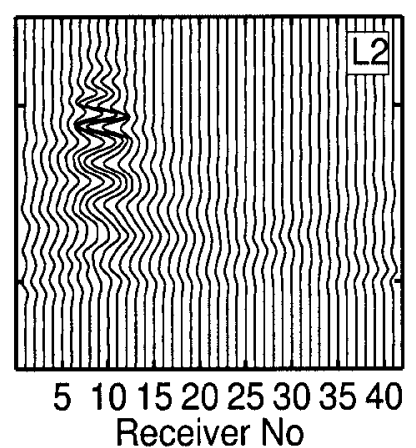

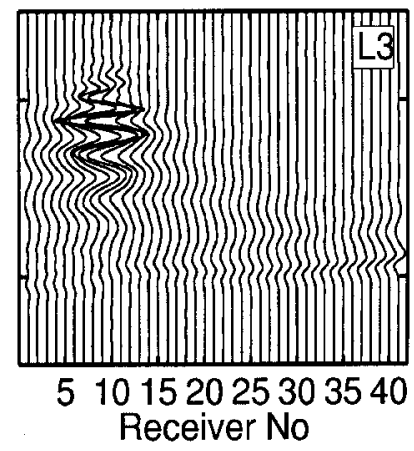

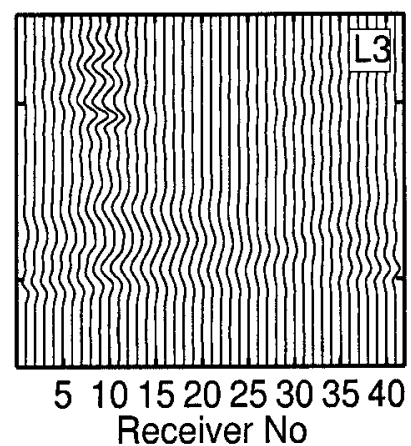

Figure 12

Top: Horizontal components of velocity across profiles L1-3 on the fault for the homogeneous simple fault structure shown in the Figure above. Bottom: Horizontal components of velocity across profiles L1-3 on the fault for the split fault model. Note that the trapping efficiency considerably decreases as the receiver profile reaches the fault discontinuity. These differential effects may help in recovering fault zone structure at depth.

As in the cases associated with Figures 9-10, if the profiles would be interpreted individually assuming continuous structures with depth, the resulting parameters would not represent the actual fault zone structure well. However, the differential information which is contained in observations from various propagation directions may help in disentangling complexity in fault zone structure at depth.

\section{Discussion}

The observation and interpretation of fault zone guided head and trapped waves may be an important tool for determining the fault zone structure at depth, with a spatial resolution not possible with other indirect seismic methods such as raytheoretical tomography. The propagation of fault zone waves for plane-parallel layered structures (e.g., BEN-ZION, 1998) as well as the nonlinear inverse procedure using analytical forward modeling tools (e.g., Michael and BEN-Zion, 1998, 2001) are well established at present. However, the effects of moderate deviations from such 
simple structures for general source-receiver geometries for 3-D models are not. To study the seismic wavefield in moderately complex fault zone structures, we use a 3-D numerical algorithm based on high-order (fourth-order space, second-order time) staggered finite-difference method. To verify the numerical method we compared numerical calculations with 3-D analytical results for point sources along a material interface (BEN-ZION, 1990, 1999). Accurate numerical calculations with staggered grids for models with sources at material interfaces require interpolations of the material constants. Furthermore, when 2-D approximations are used, conversion to point-source solutions should be carried out before modeling real data.

To clarify possible misinterpretations we simulated wavefields through models with laterally and/or horizontally discontinuous fault zone structures. Without quantitatively analyzing the results, visual inspections of the seismograms demonstrate that fault zone discontinuities may considerably alter the observed waveforms. This and the other conclusions on effects of structural irregularities are supported by numerous numerical simulations calculated by the same method with detailed analysis of the waveform behavior (JAHNKE et al., 2001). The most prominent effects are structural (dis-) continuity along the path of the propagating wavefield. Moderate geometric variations (e.g., changes in fault zone width), realistic vertical gradients or small scale scatterers have little effect on the essential character of fault zone waves. We also find that sources outside and below a shallow FZ layer can produce considerable trapped wave energy at the surface near the fault.

The result shown here demonstrate the necessity to combine inverse procedures based on simple fault zone geometries with 3-D modeling. Furthermore, trapped mode waveforms should not be analyzed individually but - if observations are available - should be jointly interpreted with observations from different sourcereceiver paths. Substantial further research is needed to answer the question of whether fault zone structure can be determined reliably from surface observations using trapped and head waves.

\section{Acknowledgments}

We gratefully acknowledge Adam Schultz for giving us access to the Cardiff computational facilities. We also thank the Leibniz Rechenzentrum in Munich for access to their supercomputers. YBZ acknowledges support from the Southern California Earthquake Center (based on NSF cooperative agreement EAR-8920136 and USGS cooperative agreement 14-08-0001-A0899).

\section{REFERENCES}

BEn-Zion, Y. (1989), The Response of Two Joined Quarter Spaces to SH Line Sources Located at the Material Discontinuity Interface, Geophys. J. Int. 98, 213-222. 
Ben-Zion, Y. (1990), The Response of Two Half Spaces to Point Dislocations at the Material Interface, Geophys. J. Int. 101, 507-528.

Ben-Zion, Y. (1998), Properties of Seismic Fault Zone Waves and their Utility for Imaging Low-velocity Structures, J. Geophys. Res. 103, 12567-12585.

Ben-Zion, Y. (1999), Corrigendum: The Response of Two Half Spaces to Point Dislocations at the Material Interface by Ben-Zion (1990), Geophys. J. Int. 137, 580-582.

BEN-ZION, Y. and AKI, K. (1990), Seismic Radiation from an SH Line Source in a Laterally Heterogeneous Planar Fault Zone, Bull. Seismol. Soc. Am. 80, 971-994.

Ben-Zion, Y. and Andrews, D. J. (1998), Properties and Implications of Dynamic Rupture Along a Material Interface, Bull. Seismol. Soc. Am. 88, 1085-1094.

Ben-Zion, Y., Katz, S., and Leary, P. (1992), Joint Inversion of Fault Zone Head Waves and Direct P Arrivals for Crustal Structure near Major Faults, J. Geophys. Res. 97, 1943-1951.

Ben-Zion, Y. and Malin, P. (1991), San Andreas Fault Zone Head Waves near Parkfield, California, Science, 251, 1592-1594.

Carcione, J. M., Kossloff, D., and Kossloff, R. (1988), Wave Propagation Simulation in a Linear Viscoelastic Medium, Geophys. J. R. astr. Soc. 95, 597-611.

Cormier, V. F. and BerozA, G. C. (1987), Calculation of Strong Ground Motion due to an Extended Earthquake Source in a Laterally Varying Structure, Bull. Seismol. Soc. Am. 77, 1-13.

Cormier, V. F. and Spudich, P. (1984), Amplification of Ground Motion and Waveform Complexities in Fault Zones: Examples from the San Andreas and Calaveras Faults, Geophys. J. R. astr. Soc. 79, 135-152.

Crase, E., Pica, A., Noble M., McDonald, J., and Tarantola, A. (1990), Robust Elastic Nonlinear Inversion: Application to Real Data, Geophysics 55, 527-538.

Dormy, E. and Tarantola, A. (1995), Numerical Simulation of Elastic Wave Propagation Using a Finite Volume Method, J. Geophys. Res. 100, 2123-2133.

Fukao, Y., Hori, S., and Ukawa, M. (1983), Seismic Detection of the Untransformed 'Basaltic' Oceanic Crust Subducting into the Mantle, Geophys. J. Roy. Astr. Soc. 183, 169-197.

Furumura, T., Kennett, B. L. N., and Takenaka, H. (1998), Parallel 3-D Pseudospectral Simulation of Seismic Wave Propagation, Geophysics 63(1), 279-288.

Graves, R. W. (1993), Modelling 3-D Site Response Effects in the Marina District Basin, San Francisco, Cal., Bull. Seismol. Soc. Am. 83, 1042-1063.

Hori, S., Inoue, H., FukaO, Y., and Ukawa, M. (1985), A Seismological Constraint on the Depth of Basaleclogite Transition in a Subducting Oceanic Crust, Nature 303, 413-415.

Hough, S. E., Ben-Zion, Y., and Leary, P. C. (1994), Fault-zone Waves Observed at the Sourthern Joshua Tree Earthquake Rupture Zone, Bull. Seismol. Soc. Am. 8, 761-767.

Huang, B. S., Teng, T.-L., and Yeh, Y. T. (1995), Numerical Modeling of Fault-zone Trapped Waves: Acoustic Case, Bull. Seismol. Soc. Am. 85, 1711-1717.

Igel, H. (1999), Wave Propagation in Spherical Sections Using the Chebyshev Method, Geophys. J. Int. 136, $559-567$.

Igel, H., Riollet, B., and Mora, P. (1995), Anisotropic Wave Propagation through Finite Difference Grids, Geophysics 60, 1203-1216.

Igel, H., Ben-Zion, Y., and Leary, P. (1997), Simulation of SH- and P-SV-wave Propagation in Fault Zones, Geophys. J. Int. 128, 533-546.

JahnKe, G., Igel, H., and Ben-Zion, Y. (2001). Three-dimensional Calculations of Seismic Fault Zone Waves in Modestly Irregular Structures, Geophys. J. Int., submitted.

Käser, M. and Igel, H. (2001), Numerical Solution of Wave Propagation on Unstructured Grids Using Explicit Differential Operators, Geophy. Prosp., in print.

Komatitsch, D., Barnes, C., and Tromp, J. (2000), Simulation of Anisotropic Wave Propagation Based upon a Spectral Element Method, Geophysics 65, 1251-1260.

Leary, P. C., LI, Y.-G., and AKI, K. (1987), Observations and Modeling of Fault Zone Fracture Anisotropy, I, P, SV, SH Travel Times, Geophys. J. Roy. Astr. Soc. 91, 461-484.

LeARY, P. C., IGel, H., and BeN-Zion, Y. (1991), Observation and modeling of fault zone trapped waves in aid of precise precursory microearthquake location and evaluation, Earthquake Prediction: State of the Art, Proceedings International Conference, Strasbourg, France, 15-18 October 1991, pp. 321-328. 
Leary, P., Igel, H., Mora, P., and Rodrigues, D. (1993), Finite-difference Simulation of Trapped Wave Propagation in Fracture Low-velocity Layers, Can. J. Expl. Geophys. 29, 31-40.

Li, Y. G. and Leary, P. C. (1990), Fault Zone Seismic Trapped Waves, Bull. Seismol. Soc. Am. 80, 12451271.

Li, Y. G., Aki, K., Adams, D., and Hasemi, A. (1994a), Seismic Guided Waves in the Fault Zone of the Landers, California, Earthquake of 1992, J. Geophys. Res. 99, 11,705-11,722.

Li. Y. G., Vidale, J. E., Aki, K., Marone, C. J. and Lee, W. H. K. (1994b), Fine Structure of the Landers Fault Zone: Segmentation and the Rupture Process, Science 265, 367-370.

Li, Y. G. and Vidale, J. E. (1996), Low-velocity Fault-zone Guided Waves: Numerical Investigations of Trapping Efficiency, Bull. Seismol. Soc. Am. 86, 371-378.

Li, Y. G., Vidale, J. E., Aki, K., Xu, F., and Burdette, T. (1998), Evidence of Shallow Fault Zone Strengthening after the 1992 M7.5 Landers, California, Earthquake, Science 279, 217-219.

Li, Y., AKI, K., VIDAle, J., and Xu, F. (1999), Fault Zone Guided Waves from Exmplosion-generated Trapped Waves, J. Geophys. Res. 104, 20257-20275.

Michael, A. J. and Ben-Zion, Y. (1998), Inverting Fault Zone Trapped Waves with a Genetic Algorithm, EOS Trans. Amer. Geophys. Union 79, F584.

Michael, A. J. and Ben-Zion, Y. (2001), Determination of Fault Zone Structure from Seismic Guided Waves by Genetic Algorithm Inversion Using a 2D Analytical Solution: Application to the Parkfield Segment of the San Andreas Fault, ms. in preparation.

Peng, Z., Ben-Zion, Y., and Michael, A. J. (2000), Inversion of Seismic Fault Zone Waves in the Rupture Zone of the 1992 Landers Earthquake for High resolution Velocity Structure at Depth, EOS Trans. Amer. Geophys. Union, 81, F1146.

Pollitz, F. F. (2001), Regional Seismic Wavefield Computation on a 3-D Heterogeneous Earth Model by Means of Coupled Normal Mode Synthesis, submitted to Pure Appl. Geophys.

Sibson, R. H. (1999), Thickness of the Seismogenic Slip Zone: Constraints from Field Geology, EOS Trans. Am. Geophys. Union 80, F727.

Tessmer, E. (1995), 3-D Seismic Modelling of General Material Anisotropy in the Presence of the Free Surface by a Chebyshev Special Method, Geophys. J. Int. 121, 557-575.

Vidale, J. E., Helmberger, D. V., and Clayton, R. W. (1985), Finite-difference Seismograms for SH Waves, Bull. Seismol. Soc. Am. 75, 1765-1782.

VIRIEux, J. (1986), P-SV Wave Propagation in Heterogeneous Media: Velocity-stress Finite-difference Method, Geophysics 51, 889-901.

(Received February 20, 2001, revised June 11, 2001, accepted June 15, 2001)

(17) To access this journal online:

(20) http://www.birkhauser.ch 